PROCEEDINGS OF THE

AMERICAN MATHEMATICAL SOCIETY

Volume 139, Number 11, November 2011, Pages 4041-4052

S 0002-9939(2011)10954-7

Article electronically published on March 28, 2011

\title{
MULTI-POINT VARIATIONS OF THE SCHWARZ LEMMA WITH DIAMETER AND WIDTH CONDITIONS
}

\author{
DIMITRIOS BETSAKOS
}

(Communicated by Mario Bonk)

\begin{abstract}
Suppose that $f$ is holomorphic in the unit disk $\mathbb{D}$ and $f(\mathbb{D}) \subset \mathbb{D}$, $f(0)=0$. A classical inequality due to Littlewood generalizes the Schwarz lemma and asserts that for every $w \in f(\mathbb{D})$, we have $|w| \leq \prod_{j}\left|z_{j}(w)\right|$, where $z_{j}(w)$ is the sequence of pre-images of $w$. We prove a similar inequality by replacing the assumption $f(\mathbb{D}) \subset \mathbb{D}$ with the weaker assumption $\operatorname{Diam} f(\mathbb{D})=$ 2. This inequality generalizes a growth bound involving only one pre-image, proven recently by Burckel et al. We also prove growth bounds for holomorphic $f$ mapping $\mathbb{D}$ onto a region having fixed horizontal width. We give a complete characterization of the equality cases. The main tools in the proofs are the Green function and the Steiner symmetrization.
\end{abstract}

\section{INTRODUCTION}

The classical lemma of Schwarz, in its standard form, appeared in a paper of Carathéodory in the first decade of the twentieth century. Since then, it has continuously been a source of inspiration for several generations of analysts and geometers. We refer to [1], [13, [17, 5] for nontechnical introductions, historical accounts, and numerous references to the extensions and variations of the Schwarz lemma.

In the present paper, we will prove some multi-point variations of the lemma. Let $\mathbb{D}=\{z \in \mathbb{C}:|z|<1\}$ be the unit disk in the complex plane $\mathbb{C}$ and suppose that $f: \mathbb{D} \rightarrow \mathbb{C}$ is a holomorphic function. If $w \in \mathbb{C}$, we denote by $\left\{z_{1}(w), z_{2}(w), \ldots\right\}$ the finite or countably infinite set of pre-images of $w$ with the convention that each pre-image is repeated as many times as its multiplicity. One of the classical extensions of the Schwarz lemma, due to Littlewood (see e.g. [11, Theorem 214], [12, p. 52]), is the following: If $f(0)=0$ and $|f(z)|<1$ for all $z \in \mathbb{D}$, then for all $w \in f(\mathbb{D}) \backslash\{0\}$,

$$
|w| \leq \prod_{j}\left|z_{j}(w)\right| .
$$

This inequality, expressed via the Nevanlinna counting function

$$
N_{f}(w):=\sum_{j} \log \frac{1}{\left|z_{j}(w)\right|}, \quad w \in \mathbb{D} \backslash\{0\}
$$

Received by the editors September 30, 2010.

2010 Mathematics Subject Classification. Primary 30C80.

Key words and phrases. Holomorphic function, Schwarz lemma, Steiner symmetrization, capacity, Green function, inner function, diameter, width.

(C)2011 American Mathematical Society Reverts to public domain 28 years from publication 
takes the form

$$
N_{f}(w) \leq \log \frac{1}{|w|}, \quad w \in \mathbb{D} \backslash\{0\} .
$$

The inequality, in this latter form, plays an important role in the theory of composition operators; see [18.

The case of equality in (1.1) or (1.2) has been studied by Lehto [10] (see also [18]): Equality holds for some $w \in \mathbb{D} \backslash\{0\}$ if and only if $f$ is an inner function. In this case, equality holds for all $w$ outside a subset of $\mathbb{D}$ having logarithmic capacity zero. Recall that an inner function is a holomorphic function $h: \mathbb{D} \rightarrow \mathbb{D}$ whose radial limits have modulus one at a.e. point of $\partial \mathbb{D}$.

Another variation of the Schwarz lemma was proved by Landau and Toeplitz (see 6], 14, Problem 239, p. 151]) and involves the diameter Diam $f(\mathbb{D})$ of $f(\mathbb{D})$. It states that if $f: \mathbb{D} \rightarrow \mathbb{C}$ is holomorphic and $\operatorname{Diam} f(\mathbb{D})=2$, then for every $0<r<1$,

$$
\operatorname{Diam} f(r \mathbb{D}) \leq 2 r
$$

and

$$
\left|f^{\prime}(0)\right| \leq 1
$$

Moreover, equality holds in (1.3) for some $0<r<1$ or in (1.4) if and only if $f(z)=$ $a+c z$ for some constants $a \in \mathbb{C}$ and $c \in \partial \mathbb{D}$. Here and below, $r \mathbb{D}=\{r z: z \in \mathbb{D}\}$.

Recently, Burckel, Marshall, Minda, Poggi-Corradini, and Ransford [6] (see also [4) proved the stronger result that under the same assumptions, the function $r \mapsto$ $\operatorname{Diam} f(r \mathbb{D}) /(2 r)$ is increasing and in fact it is strictly increasing unless $f(z)=a+c z$. Moreover, they proved a related modulus growth estimate: If $\operatorname{Diam} f(\mathbb{D})=2$, then for all $z \in \mathbb{D}$,

$$
|f(z)-f(0)| \leq \frac{2|z|}{1+\sqrt{1-|z|^{2}}} .
$$

Equality holds in (1.5) for some $z \in \mathbb{D} \backslash\{0\}$ if and only if

$$
f(z)=c \frac{z-b}{1-\bar{b} z}
$$

for some constants $a \in \mathbb{C}, b \in \mathbb{D} \backslash\{0\}, c \in \partial \mathbb{D}$. Our first theorem is a multi-point extension of (1.5).

Theorem 1. Let $f: \mathbb{D} \rightarrow \mathbb{C}$ be a holomorphic function with $\operatorname{Diam} f(\mathbb{D})=2$. Then

$$
\frac{4|w-f(0)|}{4+|w-f(0)|^{2}} \leq \prod_{j}\left|z_{j}(w)\right|, \quad w \in f(\mathbb{D}) \backslash\{f(0)\} .
$$

Equality holds in (1.6) for some $w_{o} \in f(\mathbb{D}) \backslash\{f(0)\}$ if and only if there exist an $a \in \mathbb{D} \backslash\{0\}$ and an inner function $h$ with $h(0)=0$ such that

$$
f(z)=\frac{h(z)+a}{1+\bar{a} h(z)}+a+w_{o}, \quad z \in \mathbb{D} .
$$

The paper [6] contains various other versions of the Schwarz lemma with geometric conditions involving the logarithmic capacity, the $n$-diameter, and the area 
of $f(\mathbb{D})$. We will prove an analogous result involving the horizontal width. This quantity is defined by

$$
W(f(r \mathbb{D}))=\sup _{z_{1}, z_{2} \in r \mathbb{D}}\left|\operatorname{Re} f\left(z_{1}\right)-\operatorname{Re} f\left(z_{2}\right)\right|, \quad 0<r \leq 1 .
$$

The horizontal width of the image domain was studied by Pólya and Szegö 14 , Problem 238, p. 151; Problem 289, p. 162], who showed that if $W(f(\mathbb{D}))=\frac{\pi}{2}$, then

$$
\left|f^{\prime}(0)\right| \leq 1
$$

with equality if $f(z)=\tan ^{-1} z$. Note that the function $\tan ^{-1} z$ maps $\mathbb{D}$ conformally onto the vertical strip

$$
S_{o}=\left\{w \in \mathbb{C}:-\frac{\pi}{4}<\operatorname{Re} w<\frac{\pi}{4}\right\} .
$$

Hayman [8, p. 129] gave another proof of (1.8) using Steiner symmetrization. We will also use Steiner symmetrization to prove the following theorem. Vuorinen [20, p. 143] has obtained similar results for quasiregular mappings.

Theorem 2. Let $f: \mathbb{D} \rightarrow \mathbb{C}$ be a holomorphic function with $W(f(\mathbb{D}))=\frac{\pi}{2}$.

(a) For every $r \in(0,1)$,

$$
W(f(r \mathbb{D})) \leq 2 \tan ^{-1} r .
$$

Equality holds for some $r \in(0,1)$ if and only if there exist $b \in \mathbb{C}$ and $\theta \in[0,2 \pi)$ such that $f(z)=\tan ^{-1}\left(e^{i \theta} z\right)+b$.

(b) For every $w \in f(\mathbb{D}) \backslash\{f(0)\}$,

$$
\tanh |w-f(0)| \leq \prod_{j}\left|z_{j}(w)\right| .
$$

Equality holds for some $w_{o} \in f(\mathbb{D}) \backslash\{f(0)\}$ if and only if there exists an inner function $h$ with $h(0)=0$ such that

$$
f(z)=\tan ^{-1}\left(\frac{h(z)+i u}{1-i u h(z)}\right)+t, \quad z \in \mathbb{D}
$$

with $u=\tanh \left(\operatorname{Im} w_{o}\right) \in(-1,1)$ and $t=\operatorname{Re} w_{o} \in \mathbb{R}$. If $f$ has this form, then it maps $\mathbb{D}$ onto the vertical strip

$$
S_{t}=\left\{w \in \mathbb{C}: t-\frac{\pi}{4}<\operatorname{Re} w<t+\frac{\pi}{4}\right\}
$$

and the point $f(0)$ lies on the mid-line $\{w \in \mathbb{C}: \operatorname{Re} w=t\}$ of this strip. Moreover, equality holds in (1.10) for all $w \neq f(0)$ on the mid-line of $S_{t}$.

(c) For every $z \in \mathbb{D}$,

$$
|f(z)-f(0)| \leq \tanh ^{-1}|z| .
$$

Equality holds for some $z_{o} \in \mathbb{D} \backslash\{0\}$ if and only if there exist $t \in \mathbb{R}$ and $u \in(-1,1)$ such that

$$
f(z)= \pm \tan ^{-1}\left(\frac{i e^{-i \theta_{o}} z+i u}{1+u e^{-i \theta_{o} z}}\right)+t, \quad z \in \mathbb{D},
$$

where $\theta_{o}=\arg z_{o}$. If $f$ has this form, then equality holds in (1.11) for all $z$ on the diameter $\left\{r e^{i \theta_{o}}:-1<r<1\right\}$ of $\mathbb{D}$. 
Remark. In view of Theorem 2 and of the monotonicity results in [6], one could conjecture that the function

$$
r \mapsto \frac{W(f(r \mathbb{D}))}{\tan ^{-1} r}, \quad 0<r<1,
$$

is increasing. However, the referee of the paper showed that this conjecture is false by using the function $f(z)=\tan ^{-1}(z / \rho)$, where $\rho$ is a fixed small positive number.

We will prove Theorems 1 and 2 in sections 3 and 4 after the preparatory next section.

\section{Steiner symmetrization and the Green function}

2.1. Steiner symmetrization. The Steiner symmetrization of an open set $A \subset \mathbb{C}$ with respect to a line $\ell$ is an open set $S_{\ell} A$, symmetric with respect to $\ell$. We define it by determining the intersection of $S_{\ell} A$ with every line perpendicular to $\ell$. Let $\gamma$ be such a line. Then $\gamma \cap S_{\ell} A$ is an open linear segment on $\gamma$, symmetric with respect to $\ell$ and having length equal to $m_{1}(\gamma \cap A)$; here $m_{1}$ denotes the one-dimensional Lebesgue measure. If $\gamma \cap A=\varnothing$, then $\gamma \cap S_{\ell} A=\varnothing$.

The Steiner symmetrization $S_{\ell} K$ of a compact set $K$ is defined similarly with the difference that $\gamma \cap S_{\ell} K$ is a closed segment. Also, if $m_{1}(\gamma \cap K)=0$ but $\gamma \cap K \neq \varnothing$, then, by definition, $\gamma \cap S_{\ell} K$ is the singleton $\ell \cap \gamma$. The set $S_{\ell} K$ is a compact set, symmetric with respect to the line $\ell$.

For the basic properties and applications of Steiner symmetrization in complex analysis, we refer to [7, 8]. Here we mention only two elementary properties. Suppose that $D$ is a planar domain and $\ell$ is a line. Then $S_{\ell} D$ is a simply connected domain. If $S_{\ell} D=D$, then we say that $D$ is Steiner symmetric with respect to $\ell$. The second property is that

$$
\operatorname{Diam} S_{\ell} D \leq \operatorname{Diam} D
$$

2.2. The Green function. The basic theory of the Green function is presented e.g. in [16]. The Green function for the unit disk has an explicit formula:

$$
g\left(z_{1}, z_{2}, \mathbb{D}\right)=\log \left|\frac{1-z_{1} \overline{z_{2}}}{z_{1}-z_{2}}\right| \text {. }
$$

It follows easily from this formula that

$$
g\left(z_{1}, z_{2}, \mathbb{D}\right) \geq g\left(-\left|z_{1}\right|,\left|z_{2}\right|, \mathbb{D}\right),
$$

with equality if and only if the points $z_{1}, z_{2}$ lie on the same diameter but on different radii of $\mathbb{D}$.

Suppose that $D$ is a domain in $\mathbb{C}$ possessing a Green function. Let $z_{1}, z_{2} \in$ $D, z_{1} \neq z_{2}$. Let $\ell$ be a line. Then we have (see [2, [9, Chapter 9])

$$
g\left(z_{1}, z_{2}, D\right) \leq g\left(p_{\ell}\left(z_{1}\right), p_{\ell}\left(z_{2}\right), S_{\ell} D\right)
$$

where $p_{\ell}\left(z_{j}\right)$ is the vertical projection of $z_{j}$ on $\ell, j=1,2$. Moreover (see [3. p. 416]), equality holds in (2.3) if and only if there exists a line $l$ parallel to $\ell$ such that $z_{1}, z_{2} \in l$ and $S_{l} D \stackrel{\text { n.e. }}{=} D$. The last relation means that the two sets differ on a set of zero logarithmic capacity.

We will need another result on the behavior of the Green function under symmetrization. 
Lemma 1. Let $D$ be a domain in $\mathbb{C}$ which is Steiner symmetric with respect to a line $\ell$. Let $z, \zeta \in \ell \cap D$. Let $\gamma$ be the line perpendicular to $\ell$ at the point $(z+\zeta) / 2$. Then

$$
g(z, \zeta, D) \leq g\left(z, \zeta, S_{\gamma} D\right) .
$$

Equality holds if and only if $S_{\gamma} D \stackrel{\text { n.e. }}{=} D$.

To prove Lemma 1 we need some facts about condensers and the modulus metric.

2.3. Condensers. A condenser is a pair $(D, K)$, where $D$ is a domain in $\mathbb{C}$ and $K$ is a compact subset of $D$. The capacity $\operatorname{cap}(D, K)$ of the condenser $(D, K)$ is defined via the Dirichlet integral; see [7, [8]. If $\ell$ is a line, then $\left(S_{\ell} D, S_{\ell} K\right)$ is a condenser and

$$
\operatorname{cap}\left(S_{\ell} D, S_{\ell} K\right) \leq \operatorname{cap}(D, K) .
$$

Moreover (see [19] or [15]), equality holds if and only if $S_{\ell} D \stackrel{\text { n.e. }}{=} D$ and $S_{\ell} K \stackrel{\text { n.e. }}{=} K$.

2.4. The modulus metric. Let $D$ be a domain in $\mathbb{C}$ and let $z, \zeta \in D, z \neq \zeta$. We define

$$
\mu(z, \zeta, D)=\inf _{z, \zeta \in K} \operatorname{cap}(D, K),
$$

where the infimum is taken over all curves $K$ in $D$ with $z, \zeta \in K$. This definition provides $D$ with a conformally invariant metric, called the modulus metric. We refer to 20] for the basic properties of the modulus metric. Here we are interested in its connection with the Green function.

Lemma 2. There exists a strictly decreasing function $\Phi:[0, \infty) \rightarrow[0, \infty)$ such that for every simply connected domain $D \varsubsetneqq \mathbb{C}$ and every pair $z, \zeta \in D$,

$$
\mu(z, \zeta, D)=\Phi(g(z, \zeta, D)) .
$$

Proof. By conformal invariance, we may assume that $D=\mathbb{D}$. In this case we have explicit expressions for both the modulus metric and the Green function. Indeed by [20, p. 104]

$$
\mu(z, \zeta, \mathbb{D})=\Phi_{1}(\rho(z, \zeta, \mathbb{D})),
$$

where $\rho(z, \zeta, \mathbb{D})$ is the hyperbolic distance between $z$ and $\zeta$ in $\mathbb{D}$ and $\Phi_{1}$ is a function given explicitly in [20] via elliptic integrals. Here we need only the fact that $\Phi_{1}$ is strictly increasing.

Also, by the explicit expressions of the hyperbolic distance and the Green function in $\mathbb{D}$,

$$
\rho(z, \zeta, \mathbb{D})=\Phi_{2}(g(z, \zeta, \mathbb{D})),
$$

where $\Phi_{2}$ is the strictly decreasing function given by

$$
\Phi_{2}(x)=\frac{1}{2} \log \frac{1+e^{-x}}{1-e^{-x}}, \quad x>0 .
$$

We conclude that $\mu(z, \zeta, D)=\Phi(g(z, \zeta, D))$, where $\Phi=\Phi_{1} \circ \Phi_{2}$. 
2.5. Proof of Lemma 1. Suppose that $D, \ell, z, \zeta, \gamma$ are as in Lemma 1 Since Steiner symmetrization decreases the capacity of condensers, the extremal curve in the definition of $\mu(z, \zeta, D)$ is the closed segment $[z, \zeta]$, namely

$$
\mu(z, \zeta, D)=\operatorname{cap}(D,[z, \zeta])
$$

Now we apply Steiner symmetrization with respect to $\gamma$. Note that $S_{\gamma}[z, \zeta]=[z, \zeta]$. By (2.8) and the fact that $S_{\gamma}$ decreases the capacity of condensers, we conclude that

$$
\mu(z, \zeta, D)=\operatorname{cap}(D,[z, \zeta]) \geq \operatorname{cap}\left(S_{\gamma} D,[z, \zeta]\right)=\mu\left(z, \zeta, S_{\gamma} D\right) .
$$

The inequality (2.4) follows from (2.9) and Lemma 2 .

Now suppose that we have equality in (2.4). Since $\Phi$ is strictly decreasing, it follows from (2.9) that

$$
\operatorname{cap}(D,[z, \zeta])=\operatorname{cap}\left(S_{\gamma} D,[z, \zeta]\right) .
$$

By the result of Pouliasis [15 mentioned above, we conclude that $S_{\gamma} D \stackrel{\text { n.e. }}{=} D$. The converse is obvious.

2.6. Lindelöf's principle. An inequality stronger than that of Littlewood mentioned in the introduction is (a special case of) Lindelöf's principle; see 12, Ch. III, $\S 4]$ : If $f: \mathbb{D} \rightarrow \mathbb{C}$ is holomorphic and $f(\mathbb{D})$ is a domain possessing Green function, then for $w \in f(\mathbb{D}) \backslash\{f(0)\}$,

$$
\sum_{j} \log \frac{1}{\left|z_{j}(w)\right|}=\sum_{j} g\left(z_{j}(w), 0, \mathbb{D}\right) \leq g(w, f(0), f(\mathbb{D})) .
$$

This inequality implies the well-known inequality

$$
g\left(z_{1}, z_{2}, \mathbb{D}\right) \leq g\left(f\left(z_{1}\right), f\left(z_{2}\right), f(\mathbb{D})\right), \quad z_{1}, z_{2} \in \mathbb{D} .
$$

If equality holds in (2.12) for a pair $z_{1}, z_{2} \in \mathbb{D}$, then $f$ is a conformal mapping; see [16. p. 112].

2.7. The Green function for a strip. We will need the following estimate for the Green function of the strip

$$
S_{o}=\left\{w \in \mathbb{C}:-\frac{\pi}{4}<\operatorname{Re} w<\frac{\pi}{4}\right\} .
$$

Lemma 3. Let $w_{o}, w_{1}$ be two points in the strip $S_{o}$ with $w_{o} \neq w_{1}$. Then

$$
g\left(w_{o}, w_{1}, S_{o}\right) \leq g\left(-i\left|w_{o}-w_{1}\right| / 2, i\left|w_{o}-w_{1}\right| / 2, S_{o}\right)
$$

with equality if and only if $\operatorname{Re} w_{o}=\operatorname{Re} w_{1}=0$.

Proof. We will use the hyperbolic distance $\rho\left(z, w, S_{o}\right)$ for $S_{o}$. We denote its density by $\sigma\left(w, S_{o}\right)$. Set $s=\left|w_{o}-w_{1}\right| / 2$. To prove (2.13), it suffices to show that

$$
\rho\left(w_{o}, w_{1}, S_{o}\right) \geq \rho\left(-i s, i s, S_{o}\right) .
$$

The proof of (2.14) is based on the fact that the mid-line of $S_{o}$ is a hyperbolic geodesic for $S_{o}$. By the explicit expression for the hyperbolic density $\sigma\left(w, S_{o}\right)$ (see e.g. [9, p. 684]),

$$
\sigma\left(w, S_{o}\right) \geq \sigma\left(i|w|, S_{o}\right), \quad w \in S_{o} .
$$

Because of symmetry, we may assume that $\left|\operatorname{Re} w_{o}\right| \leq\left|\operatorname{Re} w_{1}\right|$ and that $\operatorname{Re} w_{o} \geq 0$. 
Case 1. Re $w_{1}>0$.

We may assume that the points $w_{o}, w_{1}$ lie on a line through the origin. Let $q$ be the hyperbolic geodesic (in the hyperbolic geometry of $S_{o}$ ) joining $w_{o}$ to $w_{1}$. This is an analytic curve lying in the right mid-strip. By (2.15),

$$
\begin{aligned}
\rho\left(w_{o}, w_{1}, S_{o}\right) & =\int_{q} \sigma\left(w, S_{o}\right)|d w| \geq \int_{\left|w_{o}\right|}^{\left|w_{1}\right|} \sigma\left(i r, S_{o}\right) d r \\
& =\rho\left(i\left|w_{o}\right|, i\left|w_{1}\right|, S_{o}\right)=\rho\left(-i s, i s, S_{o}\right),
\end{aligned}
$$

and (2.14) is proved.

Case 2. $\operatorname{Re} w_{1}<0$.

In this case, the hyperbolic geodesic joining $w_{o}, w_{1}$ intersects the imaginary axis. We may assume that the point of intersection is the origin. By Case 1,

$$
\begin{aligned}
\rho\left(w_{o}, w_{1}, S_{o}\right) & =\rho\left(w_{o}, 0, S_{o}\right)+\rho\left(0, w_{1}, S_{o}\right) \\
& \geq \rho\left(-i\left|w_{o}\right|, 0, S_{o}\right)+\rho\left(0, i\left|w_{1}\right|, S_{o}\right) \\
& =\rho\left(-i\left|w_{o}\right|, i\left|w_{1}\right|, S_{o}\right) \\
& =\rho\left(-i s, i s, S_{o}\right),
\end{aligned}
$$

and (2.14) is proved in Case 2 as well.

\section{Proof of Theorem 1}

Let $f: \mathbb{D} \rightarrow \mathbb{C}$ be a holomorphic function with $\operatorname{Diam} f(\mathbb{D})=2$. Let $w \in$ $f(\mathbb{D}) \backslash\{f(0)\}$. By Lindelöf's principle,

$$
\sum_{j} \log \frac{1}{\left|z_{j}(w)\right|} \leq g(w, f(0), f(\mathbb{D})) .
$$

Let $\ell$ be the line passing through $f(0)$ and $w$. By the symmetrization inequality (2.3),

$$
g(w, f(0), f(\mathbb{D})) \leq g\left(w, f(0), S_{\ell} f(\mathbb{D})\right) .
$$

By Lemma 1,

$$
g\left(w, f(0), S_{\ell} f(\mathbb{D})\right) \leq g\left(w, f(0), S_{\gamma} S_{\ell} f(\mathbb{D})\right),
$$

where $\gamma$ is the line perpendicular to $\ell$ at the point $\frac{w+f(0)}{2}$.

The domain $S_{\gamma} S_{\ell} f(\mathbb{D})$ is a Steiner symmetric with respect to each of the perpendicular lines $\ell$ and $\gamma$, and also

$$
\operatorname{Diam} S_{\gamma} S_{\ell} f(\mathbb{D}) \leq \operatorname{Diam} f(\mathbb{D})=2 .
$$

It easily follows (see [4, Lemma 2]) that $S_{\gamma} S_{\ell} f(\mathbb{D})$ is contained in the disk $\Delta$ of radius 1 , centered at $\frac{w+f(0)}{2}$. By the domain monotonicity and the conformal invariance of the Green function and (2.1),

$$
\begin{aligned}
g\left(w, f(0), S_{\gamma} S_{\ell} f(\mathbb{D})\right) & \leq g(w, f(0), \Delta) \\
& =g\left(-\frac{|f(0)-w|}{2}, \frac{|f(0)-w|}{2}, \mathbb{D}\right) \\
& =\log \frac{4+|f(0)-w|^{2}}{4|f(0)-w|} .
\end{aligned}
$$

Now (1.6) follows from (3.1)-(3.4). 
Suppose that equality holds in (1.6) for some $w_{0} \in f(\mathbb{D}) \backslash\{f(0)\}$, namely,

$$
\frac{4\left|w_{o}-f(0)\right|}{4+\left|w_{o}-f(0)\right|^{2}}=\prod_{j}\left|z_{j}\left(w_{o}\right)\right| .
$$

Then equality holds (for $w=w_{o}$ ) in (3.1)-(3.4). By the equality statements for the inequalities (2.3) and (2.4) and for the domain monotonicity of the Green function,

$$
f(\mathbb{D}) \stackrel{\text { n.e. }}{=} \Delta=\left\{w \in \mathbb{C}:\left|w-\frac{f(0)+w_{o}}{2}\right|<1\right\} .
$$

We consider the linear fractional transformations

$$
\phi_{1}(w)=w-\frac{f(0)+w_{o}}{2}: \Delta \rightarrow \mathbb{D}
$$

and

$$
\phi_{2}(w)=\frac{w-\left(f(0)-w_{o}\right) / 2}{1-w\left(\overline{f(0)-w_{o}}\right) / 2}: \mathbb{D} \rightarrow \mathbb{D} .
$$

The function $h:=\phi_{2} \circ \phi_{1} \circ f$ maps $\mathbb{D}$ to $\mathbb{D}$ with

$$
h(0)=\phi_{2} \circ \phi_{1}(f(0))=\phi_{2}\left(\frac{f(0)-w_{o}}{2}\right)=0 .
$$

Moreover, for every $j$,

$$
\begin{aligned}
h\left(z_{j}\left(w_{o}\right)\right) & =\phi_{2} \circ \phi_{1}\left(f\left(z_{j}\left(w_{o}\right)\right)\right)=\phi_{2} \circ \phi_{1}\left(w_{o}\right) \\
& =\frac{w_{o}-f(0)}{1+\left|w_{o}-f(0)\right|^{2} / 4} .
\end{aligned}
$$

By (3.5) and (3.7),

$$
\left|h\left(z_{j}\left(w_{o}\right)\right)\right|=\prod_{j}\left|z_{j}\left(w_{o}\right)\right| .
$$

Hence we have equality in Littlewood's inequality (1.1) for the function $h$. By Lehto's result mentioned in the introduction, the function $h$ is inner. It follows that

$$
\begin{aligned}
f(z) & =\phi_{1}^{-1} \circ \phi_{2}^{-1} \circ h(z)=\phi_{2}^{-1}(h(z))+\frac{f(0)+w_{o}}{2} \\
& =\frac{h(z)+\left(f(0)-w_{o}\right) / 2}{1+h(z)\left(\overline{f(0)-w_{o}}\right) / 2}+\frac{f(0)+w_{o}}{2} \\
& =\frac{h(z)+a}{1+\bar{a} h(z)}+a+w_{o}, \quad z \in \mathbb{D}
\end{aligned}
$$

with $a=\frac{f(0)-w_{o}}{2}$. Note that

$$
|a|=\frac{\left|f(0)-w_{o}\right|}{2}<1,
$$

because $f(0)$ and $w_{o}$ belong to $f(\mathbb{D})$ and $\operatorname{Diam} f(\mathbb{D})=2$.

Conversely, assume that

$$
f(z)=\frac{h(z)+a}{1+\bar{a} h(z)}+a+w_{o},
$$


where $a \in \mathbb{D} \backslash\{0\}$ and $h$ is an inner function with $h(0)=0$. Then $f$ maps $\mathbb{D}$ onto a disk $\Delta$ of radius 1 , centered at $a+w_{o}$. Since $a \in \mathbb{D} \backslash\{0\}$, we have $w_{o} \in f(\mathbb{D}) \backslash\{f(0)\}$ and $f(0)=2 a+w_{o} \neq w_{o}$. Moreover, solving for $h$ in (3.10), we obtain

$$
\begin{aligned}
h\left(z_{j}\left(w_{o}\right)\right) & =\frac{f\left(z_{j}\left(w_{o}\right)\right)-a-w_{o}-a}{1-\bar{a}\left(f\left(z_{j}\left(w_{o}\right)\right)-a-w_{o}\right)} \\
& =\frac{-2 a}{1+|a|^{2}}=\frac{w_{o}-f(0)}{1+\left|w_{o}-f(0)\right|^{2} / 4} .
\end{aligned}
$$

Since $h$ is inner, Lehto's equality statement (applied to $h$ ) and (3.11) give

$$
\prod_{j}\left|z_{j}\left(w_{o}\right)\right|=\frac{\left|w_{o}-f(0)\right|}{1+\left|w_{o}-f(0)\right|^{2} / 4} .
$$

Therefore, we have equality in (1.6) for $w=w_{o}$.

\section{Proof of Theorem 2}

(a) Let $0<r<1$. There exist points $\tilde{w}_{r}, w_{r}$ on $\partial f(r \mathbb{D})$ such that

$$
W(f(r \mathbb{D}))=\operatorname{Re} w_{r}-\operatorname{Re} \tilde{w}_{r} .
$$

Let $\tilde{z}_{r}, z_{r}$ be points with $\left|\tilde{z}_{r}\right|=\left|z_{r}\right|=r$ and $\tilde{w}_{r}=f\left(\tilde{z}_{r}\right), w_{r}=f\left(z_{r}\right)$. By (2.2),

$$
\log \frac{1+r^{2}}{2 r}=g(-r, r, \mathbb{D}) \leq g\left(\tilde{z}_{r}, z_{r}, \mathbb{D}\right) .
$$

By Lindelöf's principle,

$$
g\left(\tilde{z}_{r}, z_{r}, \mathbb{D}\right) \leq g\left(\tilde{w}_{r}, w_{r}, f(\mathbb{D})\right) .
$$

Let $S_{\mathbb{R}}$ denote Steiner symmetrization with respect to the real axis and $S_{\gamma}$ denote Steiner symmetrization with respect to the vertical line intersecting $\mathbb{R}$ at the point $t:=\operatorname{Re}\left(\tilde{w}_{r}+w_{r}\right) / 2$. By the symmetrization inequalities for the Green function ((2.3) and Lemma 1),

$$
g\left(\tilde{w}_{r}, w_{r}, f(\mathbb{D})\right) \leq g\left(\operatorname{Re} \tilde{w}_{r}, \operatorname{Re} w_{r}, S_{\gamma} S_{\mathbb{R}} f(\mathbb{D})\right) .
$$

The simply connected domain $S_{\gamma} S_{\mathbb{R}} f(\mathbb{D})$ is contained in the strip

$$
S_{t}=\left\{w \in \mathbb{C}:-\frac{\pi}{4}+t<\operatorname{Re} w<t+\frac{\pi}{4}\right\} .
$$

The domain monotonicity of the Green function yields

$$
g\left(\operatorname{Re} \tilde{w}_{r}, \operatorname{Re} w_{r}, S_{\gamma} S_{\mathbb{R}} f(\mathbb{D})\right) \leq g\left(\operatorname{Re} \tilde{w}_{r}, \operatorname{Re} w_{r}, S_{t}\right) .
$$

We now use the conformal mapping tan $: S_{o} \rightarrow \mathbb{D}$ and the conformal invariance of the Green function to obtain

$$
\begin{aligned}
g\left(\operatorname{Re} \tilde{w}_{r}, \operatorname{Re} w_{r}, S_{t}\right) & =g\left(-\frac{W(f(r \mathbb{D}))}{2}, \frac{W(f(r \mathbb{D}))}{2}, S_{o}\right) \\
& =g\left(-\tan \frac{W(f(r \mathbb{D}))}{2}, \tan \frac{W(f(r \mathbb{D}))}{2}, \mathbb{D}\right) \\
& =\log \frac{1+\tan ^{2}(W(f(r \mathbb{D})) / 2)}{2 \tan (W(f(r \mathbb{D})) / 2)} .
\end{aligned}
$$

It follows from (4.2)-(4.6) that

$$
\frac{1+r^{2}}{2 r} \leq \frac{1+\tan ^{2}(W(f(r \mathbb{D})) / 2)}{2 \tan (W(f(r \mathbb{D})) / 2)} .
$$


The function $x \mapsto\left(1+x^{2}\right) /(2 x), 0<x<1$, is strictly decreasing. Therefore (4.7) implies

$$
\tan \frac{W(f(r \mathbb{D}))}{2} \leq r
$$

which is equivalent to (1.9).

Suppose that for some $r \in(0,1)$, we have equality in (1.9). Then for this $r$, we have equality in each of the inequalities (4.2)-(4.5). We will use the equality statements from section 2. Equality in (4.3) implies that $f$ is a conformal mapping and therefore $f(\mathbb{D})$ is simply connected. Equality in (4.4) implies that $f(\mathbb{D})$ is Steiner symmetric with respect to a horizontal line and with respect to $\gamma$ and that $\operatorname{Im} \tilde{w}_{r}=\operatorname{Im} w_{r}$. Equality in (4.5), together with the above facts, implies that $f(\mathbb{D})=S_{t}$. We set $b:=f(0)$. The function $g(z):=\tan (f(z)-b)$ maps $\mathbb{D}$ conformally onto itself and $g(0)=0$. Hence there exist a $\theta \in[0,2 \pi)$ such that $g(z)=e^{i \theta} z$. It follows that

$$
f(z)=\tan ^{-1}\left(e^{i \theta} z\right)+b .
$$

Conversely, if $f$ has this form for some $\theta \in[0,2 \pi)$ and some $b \in \mathbb{C}$, then it maps $\mathbb{D}$ conformally onto the strip $S_{t}$ with $t=\operatorname{Re} b$. Moreover, for $0<r<1$, the points $e^{-i \theta} r,-e^{-i \theta} r$ are mapped to the points $b-\tan ^{-1} r, b+\tan ^{-1} r$, which implies that

$$
W(f(r \mathbb{D}))=2 \tan ^{-1} r,
$$

and hence we have equality in (1.9).

(b) Let $w \in f(\mathbb{D}) \backslash\{f(0)\}$. Set $s=\frac{1}{2}\left|f(0)-w_{o}\right|$. By Lindelöf's principle, Lemma 3. the conformal invariance and the domain monotonicity of Green function,

$$
\begin{aligned}
\sum_{j} \log \frac{1}{\left|z_{j}\right|} & =\sum_{j} g\left(z_{j}, 0, \mathbb{D}\right) \leq g(w, f(0), f(\mathbb{D})) \\
& \leq g\left(-i s, i s, S_{o}\right)=g(-\tanh s, \tanh s, \mathbb{D}) \\
& =\log \frac{1+\tanh ^{2} s}{2 \tanh s}=\log \frac{1}{\tanh (2 s)} .
\end{aligned}
$$

It follows that

$$
\tanh (2 s) \leq \prod_{j}\left|z_{j}\right|
$$

which is equivalent to (1.10).

Suppose that we have equality in (1.10) for $w=w_{o} \in f(\mathbb{D}) \backslash\{f(0)\}$. Then all the inequalities in (4.9) become equalities for $w=w_{o}$. Hence $f(\mathbb{D}) \stackrel{n_{n} . e .}{=} S_{\operatorname{Re} w_{o}}$, and $f(0)$ lies on the mid-line of $S_{\operatorname{Re} w_{o}}$. Consider the function

$$
h(z)=\frac{\tan \left(f(z)-\operatorname{Re} w_{o}\right)-i \tanh (\operatorname{Im} f(0))}{1+i \tanh (\operatorname{Im} f(0)) \tan \left(f(z)-\operatorname{Re} w_{o}\right)}
$$

which maps $\mathbb{D}$ to $\mathbb{D}$ with $h(0)=0$. Moreover,

$$
\begin{aligned}
h\left(z_{j}\left(w_{o}\right)\right) & =\frac{i \tanh \left(\operatorname{Im} w_{o}\right)-i \tanh (\operatorname{Im} f(0))}{1-\tanh (\operatorname{Im} f(0)) \tanh \left(\operatorname{Im} w_{o}\right)} \\
& =i \tanh \left(\operatorname{Im} w_{o}-\operatorname{Im} f(0)\right) .
\end{aligned}
$$

Hence

$$
\left|h\left(z_{j}\left(w_{o}\right)\right)\right|=\tanh \left|w_{o}-f(0)\right|=\prod_{j}\left|z_{j}\left(w_{o}\right)\right| .
$$


This means that we have equality in Littlewood's inequality for the function $h$. By Lehto's equality statement (see the introduction), $h$ is inner. Solving for $f$ in (4.10), we obtain

$$
f(z)=\tan ^{-1}\left(\frac{h(z)+i u}{1-i u h(z)}\right)+t, \quad z \in \mathbb{D}
$$

with $u=\tanh \left(\operatorname{Im} w_{o}\right) \in(-1,1)$ and $t=\operatorname{Re} w_{o} \in \mathbb{R}$.

Conversely, assume that $f$ has the above form for some $u \in(-1,1)$, some $t \in \mathbb{R}$, and some inner function $h$ with $h(0)=0$. Then $f$ maps $\mathbb{D}$ onto $S_{t}$ with

$$
\operatorname{Re} f(0)=t, \quad \operatorname{Im} f(0)=\tanh ^{-1} u .
$$

Solving for $h$ in (4.13) we find

$$
h(z)=\frac{\tan (f(z)-t)-i u}{1+i u \tan (f(z)-t)}, \quad z \in \mathbb{D} .
$$

Using this expression for $h$ and (4.14), we infer that for every $w$ in the mid-line of $S_{t}$, namely for $\operatorname{Re} w=t$, we have

$$
h\left(z_{j}(w)\right)=i \tanh (\operatorname{Im} w-\operatorname{Im} f(0)) .
$$

Since $h$ is inner, Lehto's result (applied to $h$ ) and (4.16) imply that

$$
\prod_{j}\left|z_{j}(w)\right|=\tanh |w-f(0)| .
$$

Therefore for such a $w$, 1.10 holds with equality.

(c) The inequality (1.11) follows at once from (1.10). However, in order to treat the equality case, we give a straightforward proof. Let $z \in \mathbb{D}$. By the symmetrization argument (as in the proof of (b)),

$$
g(z, 0, \mathbb{D}) \leq g\left(f(z), f(0), S_{t}\right),
$$

where $t=\operatorname{Re} f(0)$. This is equivalent to (1.11).

Suppose that equality holds in (1.11) for some $z_{o} \in \mathbb{D} \backslash\{0\}$. This means that

$$
g\left(z_{o}, 0, \mathbb{D}\right)=g\left(f\left(z_{o}\right), f(0), S_{t}\right),
$$

where $t=\operatorname{Re} f\left(z_{o}\right)=\operatorname{Re} f(0)$. By arguments similar to those in (b), $f$ is a conformal mapping of $\mathbb{D}$ onto $S_{t}$ and the points $0, z_{o}$ are mapped on the mid-line of $S_{t}$. Simple calculations show that all such mappings have the form

$$
f(z)= \pm \tan ^{-1}\left(\frac{i e^{-i \theta_{o}} z+i u}{1+u e^{-i \theta_{o} z}}\right)+t, \quad z \in \mathbb{D},
$$

where $\theta_{o}=\arg z_{o}$ and $u=\tanh \left(\operatorname{Im} f\left(z_{o}\right)\right)$.

Conversely, if $f$ has this form for some $u \in(-1,1)$ and some $t, \theta_{o} \in \mathbb{R}$, then it maps $\mathbb{D}$ conformally onto $S_{t}$. Also, every $z$ lying on the diameter $\left\{r e^{i \theta_{o}}:-1<r<\right.$ $1\}$ of $\mathbb{D}$ is mapped on the mid-line of the strip $S_{t}$. By the conformal invariance of the Green function, for such a $z \neq 0$,

$$
g(0, z, \mathbb{D})=g\left(f(0), f(z), S_{t}\right),
$$

which is equivalent to (1.11) with equality. 


\section{REFERENCES}

1. H.P. Boas, Julius and Julia: Mastering the art of the Schwarz lemma. Amer. Math. Monthly 117 (2010), 770-785.

2. A. Baernstein II, Integral means, univalent functions and circular symmetrization. Acta Math. 133 (1974), 139-169. MR0417406 (54:5456)

3. D. Betsakos, Equality cases in the symmetrization inequalities for Brownian transition functions and Dirichlet heat kernels. Ann. Acad. Sci. Fenn. Math. 33 (2008), 413-427. MR2431373 (2009e:60175)

4. D. Betsakos, Geometric versions of Schwarz's lemma for quasiregular mappings. Proc. Amer. Math. Soc. 139 (2011), 1397-1407.

5. A.F. Beardon, D. Minda, A multi-point Schwarz-Pick lemma. J. Anal. Math. 92 (2004), 81104. MR2072742 (2005f:30044)

6. R.B. Burckel, D.E. Marshall, D. Minda, P. Poggi-Corradini, T.J. Ransford, Area, capacity and diameter versions of Schwarz's lemma. Conform. Geom. Dyn. 12 (2008), 133-152. MR2434356 (2010j:30050)

7. V.N. Dubinin, Symmetrization in the geometric theory of functions of a complex variable. Russian Math. Surveys 49 (1994), 1-79. MR.1307130(96b:30054)

8. W.K. Hayman, Multivalent Functions. Second edition. Cambridge University Press, 1994. MR:1310776 (96f:30003)

9. W.K. Hayman, Subharmonic Functions, Vol. 2. Academic Press, 1989. MR.1049148 (91f:31001)

10. O. Lehto, On the distribution of values of meromorphic functions of bounded characteristic. Acta Math. 91 (1954), 87-112. MR0062226 (15:947c)

11. J.E. Littlewood, Lectures on the Theory of Functions. Oxford University Press, 1944. MR0012121 (6:261f)

12. R. Nevanlinna, Analytic Functions. Springer, 1970. MR0279280 (43:5003)

13. R. Osserman, From Schwarz to Pick to Ahlfors and beyond. Notices Amer. Math. Soc. 46 (1999), 868-873. MR 1704258 (2000i:30049)

14. G. Pólya, G. Szegö, Problems and Theorems in Analysis. I. Springer, 1978. MR.580154 (81e:00002)

15. S. Pouliasis, Equality cases for condenser capacity inequalities under symmetrization. Preprint, 2010.

16. T. Ransford, Potential Theory in the Complex Plane. Cambridge University Press, 1995. MR1334766 (96e:31001)

17. R. Remmert, Theory of Complex Functions. Springer-Verlag, 1991. MR:1084167 (91m:30001)

18. J.H. Shapiro, The essential norm of a composition operator. Ann. of Math. (2) 125 (1987), 375-404. MR881273 (88c:47058)

19. V. A. Shlyk, A uniqueness theorem for the symmetrization of arbitrary capacitors. Siberian Math. J. 23 (1982), 267-287. MR0652233 (84i:31002)

20. M. Vuorinen, Conformal Geometry and Quasiregular Mappings. Lecture Notes in Math., 1319, Springer-Verlag, 1988. MR.950174 (89k:30021)

Department of Mathematics, Aristotle University of Thessaloniki, 54124 ThessaLONIKI, GREECE

E-mail address: betsakos@math.auth.gr 